(C) 2016 IEEE. Personal use of this material is permitted. Permission from IEEE must be obtained for all other uses, in any current or future media, including reprinting/republishing this material for advertising or promotional purposes, creating new collective works, for resale or redistribution to servers or lists, or reuse of any copyrighted component of this work in other works. 


\title{
Control Strategy for Distribution Generation Inverters to Maximize the Voltage Support in the Lowest Phase During Voltage Sags
}

\author{
Antonio Camacho, Miguel Castilla, Jaume Miret, Member, IEEE, L. García de Vicuña, \\ Miguel Andrés Garnica
}

\begin{abstract}
Voltage sags are considered one of the worst perturbations in power systems. Distributed generation power facilities are allowed to disconnect from the grid during grid faults whenever the voltage is below a certain threshold. During these severe contingencies, a cascade disconnection could start, yielding to a blackout. To minimize the risk of a power outage, inverter-based distributedgeneration systems can help to support the grid by appropriately selecting the control objective. Which control strategy performs better when supporting the grid voltage is a complex decision that depends on many variables. This paper presents a control scheme that implements a smart and simple strategy to support the fault: the maximum voltage support for the lowest phase voltage. Therefore, the faulted phase that is more affected by the sag can be better supported since this phase voltage increases as much as possible, reducing the risk of under-voltage disconnection. The proposed controller has the following features: a) maximizes the voltage in the lowest phase, b) injects the maximum rated current of the inverter, and c) balances the active and reactive power references to deal with resistive and inductive grids. The control proposal is validated by means of experimental results in a laboratory prototype.
\end{abstract}

Index Terms-Grid faults, Positive-negative sequence, Voltage dip, Voltage ride-through, Voltage sag, Voltage support

\section{INTRODUCTION}

$\mathbf{T}$ HE high integration of renewable energy sources and distributed generation (DG) [1] into the grid has changed the requirements and the operation of power systems [2]. Some years ago, DG sources represented a low contribution over the total energy market. At present, the total amount of nonconventional energy production can achieve up to $50 \%$ of the total electrical comsumption and even more [3], [4]. This new scenario presents a potential benefit over the classical one, since DG reduces energy losses and many times provides from clean and sustainable energy sources [5]. However, the massive

This work has been supported by ELAC2014/ESE0034 from the European Union and its linked Spanish national project PCIN-2015001. We also appreciate the support from the Ministry of Economy and Competitiveness of Spain and European Regional Development Fund (FEDER) under project ENE2015-64087-C2-1-R.

A. Camacho, M. Castilla, J. Miret, L. García de Vicuña and M. A. Garnica are with the Department of Electronic Engineering, Technical University of Catalonia, 08800 Vilanova i la Geltrú, Spain (e-mail: antonio.camacho.santiago@upc.edu; miquel.castilla@upc.edu; jmiret@eel.upc.edu; vicuna@eel.upc.edu; miguel.garnica@armada.mil.co) integration of those systems can compromise the reliability of the whole system, in particular during voltage sags.

Voltage sags are one of the worst perturbations in power systems characterized by a short-time voltage drop in one or several phases [6]. The main causes are short-circuits by unintentional contact, lightnings and equipment failure [7]. During the fault, DG grid-connected power sources must withstand the perturbation and operate continuously. However, in order to protect the installation, when the voltage is low for a long time, the power facility can be disconnected according to the voltage profile and trip times defined in grid codes [8][10]. To reduce the risk of disconnection, appropriate lowvoltage ride-through (LVRT) protocols need to be developed to support the grid voltages.

The conventional LVRT services [11]-[15] are focused on medium to big power plants above a certain rated power. The main objective of grid codes is to support the grid with reactive power during the fault, and to guarantee the continuous operation to avoid sudden loss of power generation. The control proposal of this paper should be understood as a part of the new generation of grid codes that could apply to any rated power generation source, that is being interfaced with the grid via flexible power converters, and tied to any type of grid impedance (inductive and resistive with high or low values).

Voltage support has become a challenging method to improve the grid reliability during grid faults. The main reason for this is the huge amount of fast reactive power provisions available in inverter-based DG systems. How to manage the current injected by the DG into the grid is the main issue for the new development of such control strategies. However, which strategy is appropriate for a given voltage sag is a complex problem to be solved. This paper presents a voltage support control scheme that implements one of the simplest objectives: to maximize the lowest phase voltage. This strategy is fairly justified by the fact that raising the lowest voltage could avoid under-voltage disconnection.

The trend in voltage support control is nonetheless dedicated to mainly inductive grids [16]-[20], while low and medium voltage grids with some inherent resistive behavior have been less discussed in the literature [21]-[24]. This study presents a control scheme that applies for any type of grid impedance, either inductive, resistive or a combination of both. Indeed, the proposed solution is also valid for weak or stiff grids since the optimal solution does not rely on the magnitude of the grid 


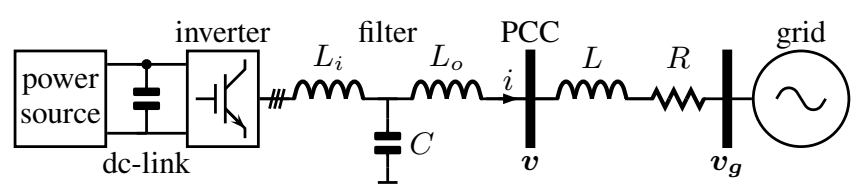

Fig. 1. Simplified scheme of a three-phase grid-connected distributed generation inverter.

impedance, but on the inductive/resistive ratio $(L / R)$.

Several works have been proposed in the literature for voltage support during faults [16]-[28]. Most of these works are based on symmetric sequences to deal with unbalanced grid voltages, which has been established as the preferred method to develop advanced voltage support controllers. The main features investigated in this research area cover: different voltage support objectives [16]-[20], [27], [28], maximum current injection during the sag [17], [19], [25], [26] and grid impedance matching [22]-[24]. However, none of these works joins these three features at the same time, i.e. improving the voltage support for any type of grid impedance while injecting the maximum rated current. To simultaneously accomplish these objectives, several issues need to be addressed first as will be shown along the work.

Compared with previous state-of-the-art control strategies, the main contributions of the proposed work are: a) the formulation of a new voltage support objective for distribution generation inverters during grid faults, b) the control scheme that guarantees the maximum increase in the lowest phase voltage, subject to the constraint of a safe current injection, and c) the flexibility of the proposal to deal with inductive and resistive, weak or stiff grids. This method has the advantage of increasing as much as possible the lowest phase voltage in a safe manner. By considering the grid impedance, it is possible to extend the voltage support range. Thus, the risk of under-voltage disconnection is reduced.

The paper is organized as follows. Section II formulates the problem. Section III solves the problem of maximizing the lowest phase voltage. Section IV deals with the impact of the grid impedance on the performance of the proposal. Section $\mathrm{V}$ presents the experimental results obtained in a laboratory prototype. Finally Section VI concludes the paper.

\section{Problem Statement}

This section starts presenting the basics of the power plant under control, then the main controller tasks are enumerated, and the voltage support concept is briefly explained, finally the control objectives and the problem to be solved are presented.

\section{A. Power Plant}

The main objective of the proposed work is to maximize the lowest phase voltage during grid faults. This proposal applies to DG inverters and/or static synchronous compensators with energy storage capacity that are interfaced with the grid via full-power converters. A generic scheme of this kind of controlled systems is presented in Fig.1. In the figure, the primary source is connected to the three-phase inverter. In
TASK : Controller $\left(t_{k}\right)$

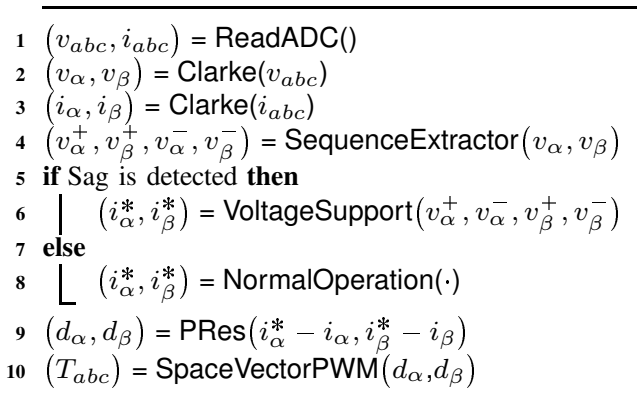

Fig. 2. Pseudo-code of the controller task.

between, a dc-link is needed to balance the power flow from the source to the grid. The main inverter's parts are the power switches and the $L C L$ output filter to reduce the switching harmonics. The inverter connects to the grid at the point of common coupling (PCC), which corresponds to the place where the phase voltages $\boldsymbol{v}$ for the different voltage support controls should be compared. The grid is modeled as a voltage source $\boldsymbol{v}_{\boldsymbol{g}}$ and an equivalent grid impedance $R$ and $L$. It should be mentioned that for developing the control proposal, the $L / R$ ratio need to be known. To this end, two main methods can be borrowed: the knowledge of the nearby elements close to the facility, or an on-line grid impedance estimator [29], [30].

In order to simplify the study, along this work it is assumed that no local load is tied to the DG inverter. By assuming this, the theoretical analysis becomes simpler and the control development can be derived in a more intuitive way.

\section{B. Controller}

After presenting the main system parts, the controller functionality is briefly reviewed. To control the plant in Fig. 1, the inverter is set in current-controlled mode. The pseudocode that implements the main controller parts is presented in Fig. 2. The controller task is activated each sampling time, the first step is to sense the voltages and currents. Then, by using Clarke transformation, the instantaneous values in the $\alpha$ - $\beta$ domain are obtained. A sequence extractor [31] is needed to decompose the voltage into the symmetric sequence counterparts $\left(v_{\alpha}^{+}\right.$and $v_{\beta}^{+}$for the positive sequence, and $v_{\alpha}^{-}$ and $v_{\beta}^{-}$for the negative one). Once the sag is detected, the proposed voltage support control is launched. This part of the code will be fully described in Section III-B. For the case where no sag has been detected, the current references are selected based on the conventional behavior of a currentmode inverter [32], depending on the production, desired power factor, operational and economic constraints. After computing the reference currents $i_{\alpha}^{*}$ and $i_{\beta}^{*}$, a proportionalresonant controller compares the references and the sensed currents to get the duty cycles of the inverter $d_{\alpha}$ and $d_{\beta}$. Finally, a space vector PWM computes the switching times of each inverter branch. 


\section{Voltage Support}

Based on the scheme in Fig. 1, the relations among the inverter and the grid voltages, the injected currents, and the grid impedance can be expressed as

$$
\boldsymbol{v}-\boldsymbol{v}_{g}=R \boldsymbol{i}+L \frac{d \boldsymbol{i}}{d t}
$$

where the voltage support from the grid to the PCC is $\Delta \boldsymbol{v}=\boldsymbol{v}-\boldsymbol{v}_{g}$. This voltage increment clearly depends on the grid impedance as will be shown along this work. Indeed, the main limitation of the voltage support control schemes is the magnitude of this impedance. For weak grids, higher voltage support effects are obtained than for stiff ones.

Also, as previously stated, in order to inject a high-quality current into the grid, and to deal with unbalanced voltages, the method of symmetric sequences has become the preferred tool to develop advanced voltage support controllers. To further develop the control, some magnitudes based on the symmetric sequence theory are required. After obtaining the instantaneous voltage sequences $v_{\alpha}^{+}, v_{\alpha}^{-}, v_{\beta}^{+}$and $v_{\beta}^{-}$, the amplitudes of the positive and negative sequences are computed as

$$
\begin{aligned}
& V^{+}=\sqrt{\left(v_{\alpha}^{+}\right)^{2}+\left(v_{\beta}^{+}\right)^{2}} \\
& V^{-}=\sqrt{\left(v_{\alpha}^{-}\right)^{2}+\left(v_{\beta}^{-}\right)^{2}} .
\end{aligned}
$$

Note that along the text, uppercase is used to indicate amplitude variables while instantaneous values are written in lowercase. Besides, the sag angle between the positive and negative sequence $\varphi$, which is needed to derive the solution, is obtained as [20]

$$
\begin{aligned}
& \cos \varphi=\frac{v_{\alpha}^{+} v_{\alpha}^{-}-v_{\beta}^{+} v_{\beta}^{-}}{V^{+} V^{-}} \\
& \sin \varphi=\frac{v_{\alpha}^{+} v_{\beta}^{-}+v_{\alpha}^{-} v_{\beta}^{+}}{V^{+} V^{-}} \\
& \varphi=\operatorname{atan} 2(\sin \varphi, \cos \varphi)
\end{aligned}
$$

where atan 2 is the two-argument arctangent function.

Thanks to the use of symmetric sequences, the inverter can be viewed (when analyzing the voltage support effects) as a current source injecting positive sequence active $I_{p}^{+}$and reactive $I_{q}^{+}$currents into the grid. This assumption is the basis for the development of the control proposal, and the derivation of these active and reactive current references are the goal to maximize the lowest phase voltage.

In order to detail the proposed voltage support concept, Fig. 3 presents the phasor diagram of the phase voltages for a given voltage sag. The amplitude of the phases $V_{a}, V_{b}$ and $V_{c}$ is given in per unit (p.u.). As shown in the phasor graph, phase A barely suffers the sag while phase $\mathrm{C}$ (in blue color) is the most affected voltage. In the interest of clarity, the angle of phase $\mathrm{C}$ (the lowest voltage) is selected to be $180^{\circ}$. Under this consideration, the currents decomposition and the associated angles are better appreciated in the graph.

In order to raise as much as possible the lowest phase voltage, two requirements are needed, the first one is to inject the maximum rated current of the inverter through this phase (i.e. $I_{c}=I_{\max }$ ), and the second one is that this phase current

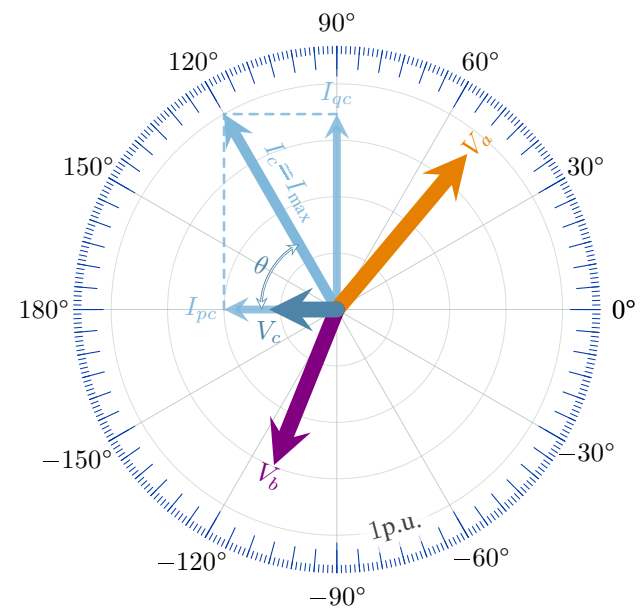

Fig. 3. Voltage support concept.

must be injected in a particular angle with respect to the phase voltage. This angle is represented by $\theta$ (which has been arbitrarily selected to $60^{\circ}$ in the phasor diagram). The first requirement allows for a safe operation and a better utilization of the inverter capabilities during the sag. The second requirement is the key point to maximize the voltage support. Without loss of generality, this injected current can be decomposed into an active $I_{p c}$ and a reactive component $I_{q c}$ which are in-phase and in-quadrature with respect to the phase voltage respectively. Depending on the magnitudes of these two currents, $\theta$ will be modified and different voltage variations will be produced depending on the grid impedance. Assuming a mainly inductive grid, it is clear that injecting a mainly reactive current $I_{q c}$ will produce better voltage support effects on the grid. In such a case $\theta \approx 90^{\circ}$. On the contrary, for a mainly resistive grid, the injected current should be mainly active $I_{p c}$, and $\theta \approx 0^{\circ}$. In between these two extreme cases, the best voltage support solution for any grid impedance must be obtained, as it will be shown in Section III-A.

\section{Control Objectives}

Along this work, the control to be implemented during the sag is based on the transformation of the unbalanced voltages into the symmetric sequences. Thus, the phase currents $I_{p c}$ and $I_{q c}$ need to be transformed into the corresponding $I_{p}^{+}$ and $I_{q}^{+}$values, consisting in the in-phase and in-quadrature currents following the positive sequence voltage $V^{+}$instead of the phase voltage $V_{c}$. The current reference generator for the symmetric sequences that achieves this objective can be expressed as [19]

$$
\begin{aligned}
i_{\alpha}^{*} & =\frac{I_{p}^{+}}{V^{+}} v_{\alpha}^{+}+\frac{I_{q}^{+}}{V^{+}} v_{\beta}^{+} \\
i_{\beta}^{*} & =\frac{I_{p}^{+}}{V^{+}} v_{\beta}^{+}-\frac{I_{q}^{+}}{V^{+}} v_{\alpha}^{+}
\end{aligned}
$$

where it can be seen how the reference currents $i_{\alpha}^{*}$ and $i_{\beta}^{*}$ cause the inverter behave as a positive sequence active and reactive current source.

It should be highlighted that the proposal deals with positive sequence voltages, being the negative sequence uncontrolled. 
This facts allows for a simpler and easier implementation, producing balanced injected currents, which is of interest during unbalanced voltage sags. As a drawback, voltage imbalance cannot be mitigated with the proposed method.

Along this work, the lowest phase voltage will be denoted as $V_{x}$ in order to deal with any voltage sag. For this purpose, the following is defined

$$
V_{x}=\min \left\{V_{a}, V_{b}, V_{c}\right\}
$$

Resuming the main control goals, i.e.

1) maximize the lowest phase voltage $V_{x}$

2) inject the maximum current of the inverter $I_{\max }$, the problem can be formulated as

$$
\begin{array}{cl}
\max _{x \in\{a, b, c\}} & V_{x}\left(I_{p}^{+}, I_{q}^{+}\right) \\
\text {subject to: } & I_{x}=I_{\max } .
\end{array}
$$

The solution relies on finding a combination of active and reactive currents $\left(I_{p}^{+}, I_{q}^{+}\right)$that simultaneously maximizes the lowest phase voltage by injecting the maximum permissible current of the inverter $I_{\max }$. As can be inferred from (10), the optimal solution comprises several stages. First, to evaluate the voltage support effect of an injected phase current into a generic $R$ - $L$ grid for any given voltage sag. Then, to find the current to voltage angle that maximizes the voltage support. And finally, to translate these expressions from the phase values into the symmetric sequence values. These three steps are fundamental to develop the solution to (10) as will be shown in next Section, and constitutes the main contributions of this work.

\section{Problem SOLUtion}

This section will be devoted to solve (10). The solution is developed in two steps: firstly, the active and reactive phase currents that maximizes the lowest phase voltage are obtained and, secondly, the positive sequence active and reactive currents are derived.

\section{A. Maximization of the Lowest Phase Voltage}

Proposition 1. Let $I_{p x}$ be the active phase current associated with the lowest phase voltage, and let $I_{q x}$ be the reactive phase current. Under this consideration, by selecting

$$
\begin{aligned}
& I_{p x}=I_{\max } \frac{R}{\sqrt{R^{2}+(\omega L)^{2}}} \\
& I_{q x}=I_{\max } \frac{\omega L}{\sqrt{R^{2}+(\omega L)^{2}}}
\end{aligned}
$$

then the lowest phase voltage $V_{x}$ is maximized and the injected phase current equals the rated current of the inverter $I_{x}=$ $I_{\max }$, for a given grid frequency $\omega$, and for any grid impedance $R$ and $L$.

Proof. The optimal active and reactive phase currents that maximize the voltage in the lowest phase are obtained by using the method of the Lagrange multipliers. The problem consists in building the Lagrange function $\mathcal{L}$ based on the objective function $f$ and the restriction $g$ so that

$$
\mathcal{L}\left(y_{1}, y_{2}, \lambda\right)=f\left(y_{1}, y_{2}\right)+\lambda g\left(y_{1}, y_{2}\right)
$$

where $\lambda$ is the Lagrange multiplier, and $y_{1}, y_{2}$ are the dependent variables.

Using (1) and the graphical projection of each phasor as shown in Fig. 3, the voltage support effects can be quantified. To this end, the phase current is decomposed into the active and reactive parts. Arranging the involved terms into a single equation, and after some mathematical manipulations, the theoretical amplitude of the phase voltage at the PCC side can be obtained. As a result, the PCC voltage is related to the voltage amplitude at the grid side, the injected currents and the grid impedance as

$$
V_{x}=\omega L I_{q x}+R I_{p x}+\sqrt{\left(V_{g x}\right)^{2}-\left(\omega L I_{p x}-R I_{p x}\right)^{2}}
$$

where $V_{x}$ is the amplitude of the supported phase voltage, $V_{g x}$ is the phase voltage at the grid side, and $I_{p x}$ and $I_{q x}$ are the unknown variables.

Once the objective function to be maximized $f\left(y_{1}, y_{2}\right)=$ $V_{x}\left(I_{p x}, I_{q x}\right)$ presented in (14) is derived, a constraint should be included to keep the inverter currents safely controlled. Resuming the current in phase $\mathrm{C}$ from Fig. 3, the restriction is straightforward since both components in the phase current $I_{p x}$ and $I_{q x}$ are $90^{\circ}$ delayed. Thus, the constraint can be written as

$$
g\left(y_{1}, y_{2}\right)=\sqrt{\left(I_{p x}\right)^{2}+\left(I_{q x}\right)^{2}}-I_{\max }=0 .
$$

Each involved term in the Lagrange function (13) has been identified for the problem stated. To obtain the optimal solution, the next step is the computation of the gradient with respect to the involved variables $I_{p x}, I_{q x}$ and $\lambda$. Finally, solving the gradient to zero gives the optimal solution

$$
\nabla \mathcal{L}\left(y_{1}, y_{2}, \lambda\right)=\left(\frac{\partial \mathcal{L}}{\partial I_{p x}}, \frac{\partial \mathcal{L}}{\partial I_{q x}}, \frac{\partial \mathcal{L}}{\partial \lambda}\right)=0 .
$$

Developing (16), the optimal currents $\left(I_{p x}, I_{q x}\right)$ that maximizes the lowest phase voltage are (11) and (12).

Alternatively, by using trigonometric identities, (11) and (12) can be rewritten as

$$
\begin{aligned}
& I_{p x}=I_{\max } \cos \theta \\
& I_{q x}=I_{\max } \sin \theta
\end{aligned}
$$

where

$$
\theta=\operatorname{atan} 2(\omega L, R)
$$

\section{B. Phase Currents to Symmetric Sequence Currents}

Proposition 2. Let $\bar{\varphi}$ be the rotation angle that translates the optimal active $I_{p x}$ and reactive $I_{q x}$ phase currents in (17) and (18) to the symmetric sequence counterparts $I_{p}^{+}$and $I_{q}^{+}$. Under such consideration the active and reactive current 
references in (7) and (8), being the optimal solution to (10), are

$$
\begin{aligned}
I_{p}^{+} & =I_{\max } \cos (\theta-\bar{\varphi}) \\
I_{q}^{+} & =I_{\max } \sin (\theta-\bar{\varphi}) .
\end{aligned}
$$

Proof. The proof is derived from trigonometric relations among the phasors analyzed in Fig. 3. Without loss of generality, it can be assumed that the phase to current angle, namely $\varphi_{V_{x}}^{I_{x}}$, equals the impedance angle, i.e.

$$
\varphi_{V_{x}}^{I_{x}}-\theta=0 .
$$

By definition,

$$
\varphi_{V_{x}}^{I_{x}}=\varphi_{V_{x}}-\varphi_{I_{x}}
$$

is the phase to current angle. Based on the phasor analysis, and conveniently developing the expressions for the voltage angle $\varphi_{V_{x}}$ and the current angle $\varphi_{I_{x}}$ as a function of the positive and negative sequences, the following relation hold [33]

$$
\varphi_{I_{x}}=-\varphi+\varphi_{x}+\operatorname{atan} 2\left(I_{q}^{+}, I_{p}^{+}\right)
$$

and the angle of the lowest phase voltage can be derived by using different methods, the proposed one decomposes the phase voltage into direct and quadrature projections, based on the positive, negative and sequence angle as

$$
\varphi_{V_{x}}=\operatorname{atan} 2\left(\frac{V^{+} \sin \left(\varphi+\varphi_{x}\right)+V^{-} \sin \left(-\varphi_{x}\right)}{V^{+} \cos \left(\varphi+\varphi_{x}\right)+V^{-} \cos \left(-\varphi_{x}\right)}\right)
$$

where $\varphi_{x}$ indicates the angle $\{0,-2 \pi / 3,+2 \pi / 3\}$ associated to the lowest phase voltage as

$$
\varphi_{x}:=\left\{\begin{array}{crl}
0, & 120^{\circ} \leqslant \varphi<240^{\circ} & \Rightarrow x=a \\
-2 \pi / 3 & 0^{\circ} \leqslant \varphi<120^{\circ} & \Rightarrow x=b \\
+2 \pi / 3 & 240^{\circ} \leqslant \varphi<360^{\circ} & \Rightarrow x=c .
\end{array}\right.
$$

Equation (24) relates the phase current angle $\varphi_{I_{x}}$ with the amplitude of the positive sequence active $I_{p}^{+}$and reactive $I_{q}^{+}$ currents. This relation is the starting point for the definition of $\bar{\varphi}$ as will be shown below.

Equation (25) is derived from the trigonometric analysis of the voltage phasors in Fig. 3, and it presents a useful method to compute phase voltage angles $\varphi_{V_{a}}, \varphi_{V_{b}}$ or $\varphi_{V_{c}}$, based on the symmetric sequence amplitudes and the sag angle.

Lastly, (26) presents a method to track the lowest phase voltage based on the sag angle in (6). Due to the properties of symmetric sequences, the sag angle itself can be used to identify the lowest phase voltage as detailed above. To achieve this goal, it is required to use the two-argument arctangent function, in brief atan2(.) as previously stated in (6). This function helps to obtain the angle in any of the four possible quadrants, between the positive and the negative sequence voltages. Thus, allowing for the identification of the lowest phase voltage [20]. The idea behind this track angle is that general expressions can be particularized by using its value $\varphi_{x} \in\{0,-2 \pi / 3,+2 \pi / 3\}$.

Using (26) in (24) and (25), and developing (23) with (22), the following holds

$$
\bar{\varphi}=\varphi_{V_{x}}-\varphi_{x}-\varphi
$$

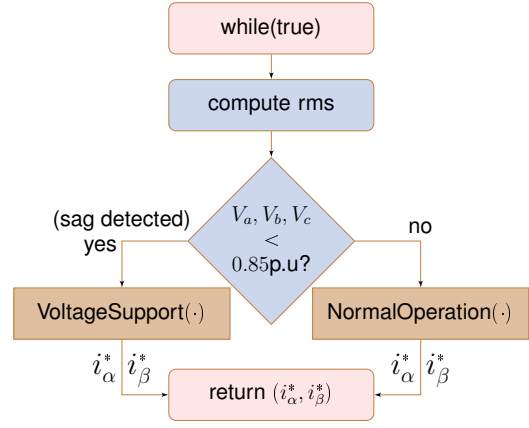

Fig. 4. Flowchart for triggering the voltage support control scheme.

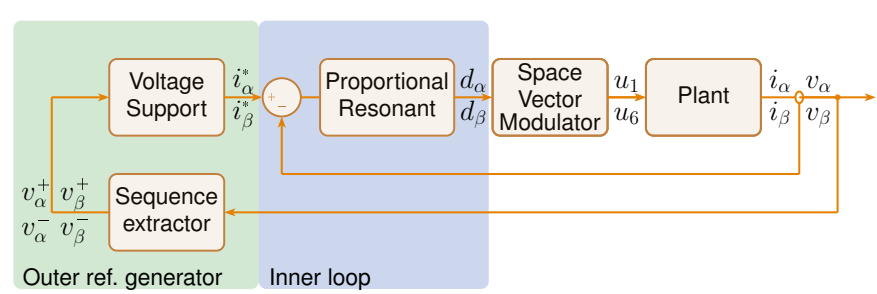

Fig. 5. Control diagram for the proposed control scheme.

which defines the rotation angle in (20)-(21) that constitutes the optimal reference currents.

Based on (11) and (12), and applying trigonometric identities to (20) and (21), the above expressions can be rewritten in terms of a rotating transformation as

$$
\begin{aligned}
& I_{p}^{+}=I_{p x} \cos \bar{\varphi}+I_{q x} \sin \bar{\varphi} \\
& I_{q}^{+}=I_{q x} \sin \bar{\varphi}-I_{p x} \sin \bar{\varphi}
\end{aligned}
$$

which conclusively is the optimal solution to (10).

After developing the theoretical solution, (28) and (29), to the problem in (10), a step by step procedure to implement this solution is presented. The flowchart for the decision algorithm is presented in Fig. 4. In this chart, it can be shown how the triggering decision for the whole voltage support controller is implemented. The trigger consists on the comparison of the root mean square (rms) voltages with the common threshold voltage of 0.85 p.u. [8], [10]. Whenever the rms voltage of one of the phases falls below this threshold, the sag is detected. Depending on this comparison, the inverter operates in normal mode or launchs the voltage support control scheme presented in Fig. 5. In this scheme, it can be appreciated the outer reference generator, the inner current loop, the modulator and the plant. The detailed implementation of the reference generator is shown in Fig. 6, which presents the algorithm that solves the problem at run-time.

\section{IMPACT OF IMPEDANCE MISMATCHES}

This Section derives an expression to quantify the deviations produced in the amplitude of the minimum voltage due to the mismatch in the grid impedance estimation.

Along the work, it has been assumed that the grid impedances $R$ and $L$ are known. Two methods have been proposed to notify the grid values: a known grid model 
FUNC $:\left(i_{\alpha}^{*}, i_{\beta}^{*}\right)=$ VoltageSupport $\left(v_{\alpha}^{+}, v_{\alpha}^{-}, v_{\beta}^{+}, v_{\beta}^{-}\right)$

$$
\begin{aligned}
& 1 \theta=\operatorname{atan2}(\omega L, R) /{ }^{*} \text { From known model or estimator */ } \\
& 2 I_{p x}=I_{\max } \cos (\theta) \\
& 3 I_{q x}=I_{\max } \sin (\theta) \\
& 4 V^{+}=\sqrt{\left(v_{\alpha}^{+}\right)^{2}+\left(v_{\beta}^{+}\right)^{2}} \\
& 5 V^{-}=\sqrt{\left(v_{\alpha}^{-}\right)^{2}+\left(v_{\beta}^{-}\right)^{2}} \\
& 6 \cos (\varphi)=\frac{v_{\alpha}^{+} v_{\alpha}^{-}-v_{\beta}^{+} v_{\beta}^{-}}{V^{+} V^{-}} \\
& 7 \sin (\varphi)=\frac{v_{\alpha}^{+} v_{\beta}^{-}+v_{\alpha}^{-} v_{\beta}^{+}}{V^{+} V^{-}} \\
& 8 \varphi=\operatorname{atan} 2(\sin (\varphi), \cos (\varphi)) \\
& 9 \text { if } 120^{\circ} \leqslant \varphi<240^{\circ} \text { then } \\
& 10 \text { L } \varphi_{x}=0 / /^{*} \text { Lowest phase is } A * \\
& 11 \text { else if } 0^{\circ} \leqslant \varphi<120^{\circ} \text { then } \\
& 12 \mathrm{~L} \varphi_{x}=-2 \pi / 3 /^{*} \text { Lowest phase is } \mathrm{B} \text { */ } \\
& 13 \text { else if } 240^{\circ} \leqslant \varphi<360^{\circ} \text { then } \\
& 14 \quad \text { L } \varphi_{x}=2 \pi / 3 /{ }^{*} \text { Lowest phase is } C \text { */ } \\
& 15 \varphi_{V_{x}}=\operatorname{atan} 2\left(\frac{V^{+} \sin \left(\varphi+\varphi_{x}\right)+V^{-} \sin \left(-\varphi_{x}\right)}{V^{+} \cos \left(\varphi+\varphi_{x}\right)+V^{-} \cos \left(-\varphi_{x}\right)}\right) \\
& 16 \bar{\varphi}=\varphi_{V_{x}}-\varphi_{x}+\varphi \\
& 17 I_{p}^{+}=I_{p x} \cos \bar{\varphi}+I_{q x} \sin \bar{\varphi} \\
& 18 I_{q}^{+}=I_{q x} \cos \bar{\varphi}-I_{p x} \sin \bar{\varphi} \\
& 19 i_{\alpha}^{*}=\frac{I_{p}^{+}}{V^{+}} v_{\alpha}^{+}+\frac{I_{q}^{+}}{V^{+}} v_{\beta}^{+} \\
& 20 i_{\beta}^{*}=\frac{I_{p}^{+}}{V^{+}} v_{\beta}^{+}-\frac{I_{q}^{+}}{V^{+}} v_{\alpha}^{+} \\
& 21 \text { return }\left(i_{\alpha}^{*}, i_{\beta}^{*}\right)
\end{aligned}
$$

Fig. 6. Pseudo-code for the controller implementation of the voltage support proposal.

based on the nearby elements where the power facility is connected, and an on-line impedance estimator. Both methods have inherent benefits and drawbacks. Whatever the method used, a certain discrepancy between the real grid values $R$ and $L$, and the estimated values used to implement the proposal $\widehat{R}$ and $\hat{L}$ could exist.

Assuming that $I_{p}^{+}$and $I_{q}^{+}$in (28)-(29) are built based on a perfect impedance estimation, the following holds

$$
V_{x}^{*}=V_{g x}+I_{\max } \sqrt{R^{2}+(\omega L)^{2}}
$$

where $V_{x}^{*}$ is the maximum voltage at the PCC that can be achieved with the proposed control scheme for the lowest phase voltage. However, in case of discrepancies between the real and estimated impedance values (i.e. $\widehat{R} \neq R$ and $\widehat{L} \neq L$ corresponding to an estimated impedance angle $\hat{\theta} \neq \theta$ ), the lowest phase voltage is

$V_{x}=\sqrt{V_{g x}^{2}-I_{\max }^{2}(R \sin \hat{\theta}-\omega L \cos \hat{\theta})^{2}}+I_{\max }(R \cos \hat{\theta}+\omega L \sin \hat{\theta})$

which correspond to a lower voltage support compared with the case where the impedance is perfectly known. The above expressions can help to further understand and quantify the effects of a poor grid impedance estimation.

It is worth mentioning that the grid model used along this work, made by a voltage source and an equivalent grid impedance, (see Fig. 1 for details), is a simplified version of
TABLE I

SYSTEM PARAMETERS

\begin{tabular}{lcc}
\hline \hline & Symbol & Nominal value \\
\hline base power & $S_{\text {base }}$ & $2.3 \mathrm{kVA}$ \\
rated current & $I_{\max }$ & $10 \mathrm{~A}($ peak $)$ \\
grid voltage & $v_{g}$ & $155 \mathrm{~V}(1-\mathrm{n}$, peak) \\
grid frequency & $\omega$ & $2 \pi 60 \mathrm{rad} / \mathrm{s}$ \\
grid inductance & $L$ & $5 \mathrm{mH}$ \\
grid resistance & $R$ & $1.3 \Omega$ \\
impedance angle & $\theta$ & $55 \circ$ \\
dc-link voltage & $V_{d c}$ & $350 \mathrm{~V}$ \\
inverter inductance & $L_{i}$ & $5 \mathrm{mH}$ \\
filter capacitor & $C$ & $1.4 \mu \mathrm{F}$ \\
output inductance & $L_{o}$ & $2 \mathrm{mH}$ \\
switching frequency & $f_{s}$ & $10 \mathrm{kHz}$ \\
\hline
\end{tabular}

the real grid. In a real power system, AC grid parameters and loads are changing dynamically. Thus, a certain discrepancy between the real and the estimated grid impedance could exists. However, as will be shown in next Section, a perfect grid impedance estimation is not really needed.

\section{EXPERIMENTAL RESUlts}

Based on Fig. 1, a scale-down laboratory prototype has been built to check the dynamic and static performance of the control proposal against a time-varying voltage sag.

\section{A. Laboratory Setup}

The experimental setup is made up of an Amrel DC power source, a Guasch three-phase inverter and a Pacific AC power source to get repetitive voltage sags. The controller task is implemented on a F28M36 Texas Instruments digital signal processor based on the pseudo-code presented in Fig. 2.

The main system parameters are collected in Table I. From this table, the nominal values for the grid impedance are $R=1.3 \Omega$ and $L=5 \mathrm{mH}$, being $\omega=2 \pi 60 \mathrm{rad} / \mathrm{s}$. Hence, the impedance angle is $\theta=\operatorname{atan} 2(\omega L, R)=55^{\circ}$. It is worth mentioning that the per unit impedance values are $R=0.08$ p.u. and $L=0.12$ p.u, which can be considered as a weak grid. However, the merit of the control scheme does not rely on the stiffness or weakness of the grid but in the $L / R$ ratio. This weak grid test-case has been selected to highlight the voltage support effects and to test the dynamic performance of the control scheme in a worst case scenario.

\section{B. Evaluation of Dynamic Performance}

The test scenario is divided into three parts, firstly the normal operation mode, in which the inverter injects $P=2000 \mathrm{~W}$ and $Q=0 \mathrm{VAr}$ into the grid. Second case corresponds to the occurrence of the voltage sag, where the proposed voltage support described in Fig. 6 is launched and the results are presented below. Finally, the third case is the post-fault state, where the inverter comes back to the normal operation mode once the fault has been cleared.

The sag under test is presented in Fig. 7. The instantaneous phase voltages are shown in the top, and the rms values are plotted in the bottom. At $t=0 \mathrm{~s}$, the sag occurs and, at $t=0.2 \mathrm{~s}$, 


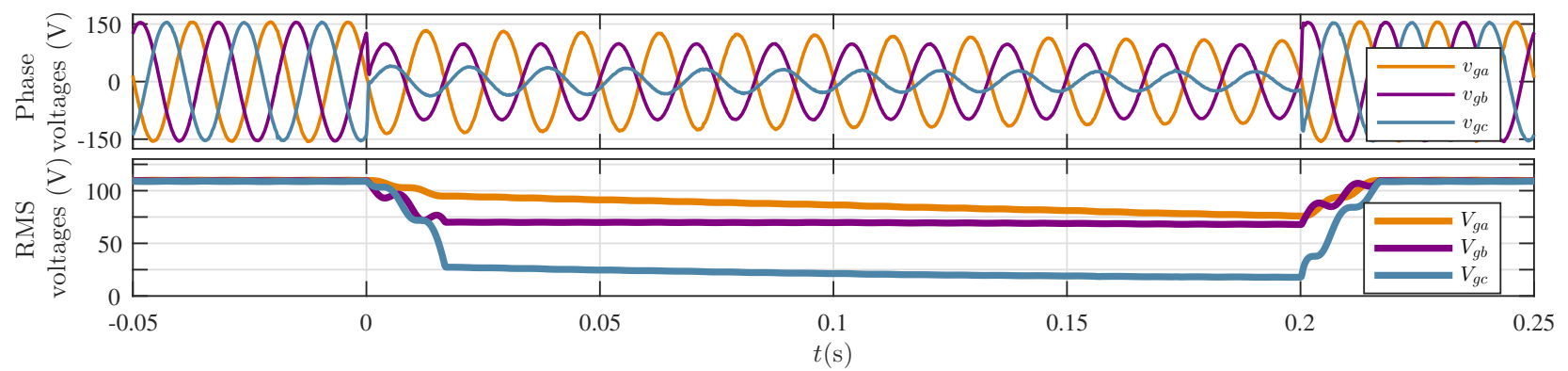

Fig. 7. Experimental results of the instantaneous and rms phase voltages for the sag under test without voltage support.

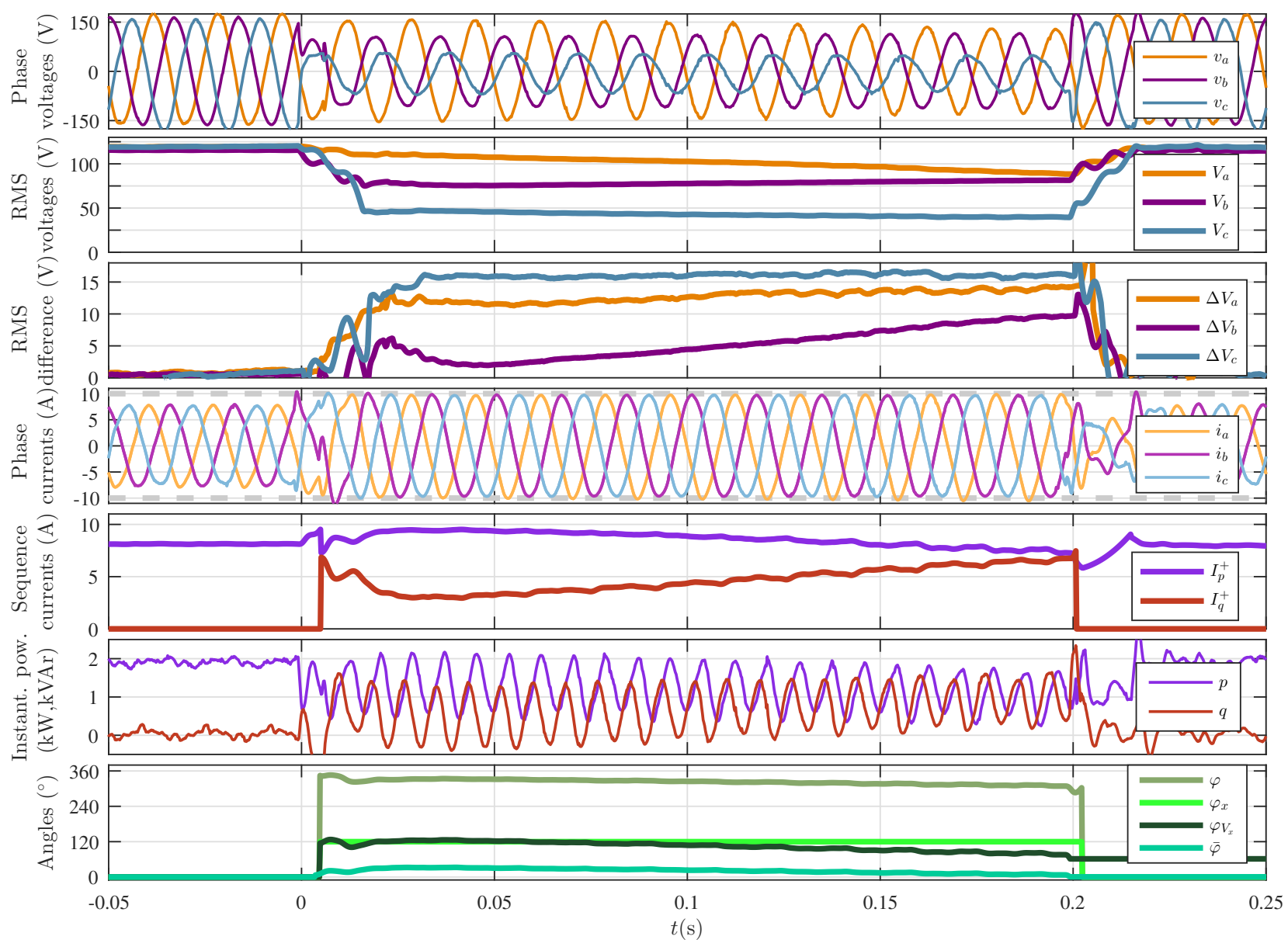

Fig. 8. Experimental results for the dynamic performance of the voltage support proposal. From top to bottom: instantaneous phase voltages, rms voltages, rms voltage difference among no voltage support and the proposed method, injected phase currents, active and reactive reference currents, instantaneous active and reactive powers, and involved angles for the computation of the solution.

the fault is cleared by the protection devices. In between, the sag has a dynamic profile to test the proposal against this complex fault. It can be seen that along the test, the threephase voltages are affected, however the lowest voltage $V_{x}$ concerns to phase $\mathrm{C}$.

In order to test the performance of the voltage support control, Fig. 8 presents the most important signals and magnitudes involved. From top to bottom, the plot shows the instantaneous phase voltages, the rms phase voltages, the rms voltage difference between the proposed control and the base-test without voltage support, the injected phase currents, the references for the active and reactive sequence currents, instantaneous active and reactive powers, and the angles as computed within the control loop.

Comparing instantaneous and rms phase voltages in Fig. 7 (without voltage support), and the voltages on top of Fig. 8 (with voltage support), it is clearly manifested the main contribution of this work: the voltage increase of the lowest phase voltage. The rms comparison of both tests can be better appreciated in the next plot, where the rms voltage difference with and without voltage support is presented. According to (30), the lowest phase raises approximately $15 \mathrm{~V}$ independently of the voltage sag profile. This is due to the fact that the control proposal computes the optimal solution based on the rotation angle as previously detailed. It should be pointed out that this high voltage increment is due to the weak grid 
scenario. However, for stiff grids, the objective of reducing the risk of under-voltage disconnection will be also accomplished although the voltage variation will be less than in the weak case. The other phases present less voltage increment, as expected due to the fact that the proposal is intended to optimize the lowest phase voltage.

Next plot of Fig. 8 presents the instantaneous phase currents that the inverter injects during the test. Before the sag and when the sag is cleared, the inverter injects an active power according to the production of the power source. When the sag is detected, the inverter launches the voltage support control to improve the voltage profile. As can be shown in this graph, the injected currents during the sag reach the rated current of the inverter ( $I_{\max }=10 \mathrm{~A}$ along the experiments). Therefore, the second objective of the proposal, which consists in a safe current injection during the sag is also accomplished. This second objective not only allows for a safe inverter operation, but also helps to take advantage of the fast reaction of inverterbased power converters when interfaced with the grid in front of sudden perturbations.

After presenting the injected phase currents, it is worth showing the references for the active and reactive sequence currents. Next plot of Fig. 8 shows the values of these two magnitudes. The active current injected before and after the sag is approximately $8 \mathrm{~A}$, and the reactive current is zero. However these values change during the sag so as to generate the appropriate current references that are needed to achieve the above mentioned control objectives. The dynamic evolution of these reference currents are due to the dynamic profile of the voltage sag.

Next plot in the figure shows the instantaneous active and reactive powers during the experiment. Before the sag, the inverter injects $2000 \mathrm{~W}$ and 0VAr, during the sag these values evolve accordingly to dynamically adapt the references to the optimal solution. Note that the energy storage capacity of the inverter allows to regulate the excess/lack of active power to the dynamic needs during the fault. When the sag has been cleared, the pre-fault values are kept. As can be appreciated in the plot, during the sag both magnitudes have oscillations at twice the grid frequency, as expected for unbalanced voltage sags. It should be noticed that the proposed method can neither reduce the voltage imbalance nor cancel the active power oscillations, which have been selected in other studies as two interesting objectives during faults. This limitation is due to the injection of positive sequence powers only, being the negative sequence unaffected. As a result, problems derived from the oscillations in the dc-link voltage should be considered, and over-voltage in the phase(s) that do not suffer the sag can be produced.

Last plot in Fig. 8 is intended to present the angles computed within the controller. These angles only have a physical meaning during the sag, so the analysis is focused on this interval. From top to bottom, the sag angle $\varphi$ presents the angle between the positive and negative sequence voltages. The track angle $\varphi_{x}$ indicates which phase is the lowest one. As can be observed in the sag voltages, the lowest phase is $V_{c}$, thus the track angle is $120^{\circ}$ during the grid fault. The phase voltage angle $\varphi_{V_{x}}$ shows where this voltage is located. Although it
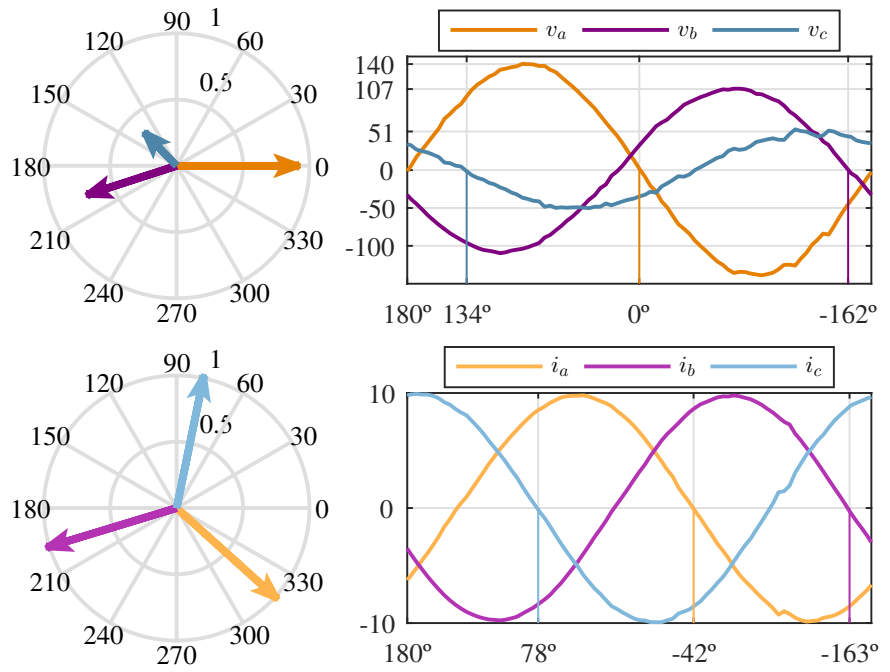

Fig. 9. Time-domain and phasor analysis of voltages and currents.

is close to $120^{\circ}$, the dynamic evolution of the voltages during the sag, makes this voltage angle to change slightly depending also on the voltage sag programmed in the experiment. Finally, the angle $\bar{\varphi}$ shows the information for the rotation from phase currents to sequence currents as theoretically explained before.

\section{Evaluation of Static Performance}

Once the dynamic performance of the controller has been presented and discussed, a static analysis has been performed to show additional features of the proposal. Fig. 9 shows the supported voltages and the injected currents in one grid cycle. The values have been arbitrary collected in the middle of the sag at time $t=0.1 \mathrm{~s}$. The figure is divided into 2 parts corresponding to the time-domain values (on the right) and the phasor plots (on the left). In order to corroborate that the voltage to current angle match the impedance angle $\theta$, the time-domain plots have labels indicating the angle instead of the time. For simplicity it has been assumed a zero angle for the voltage of phase A when crossing the origin. As it can be seen in the phasor diagram, the three phase voltages are unbalanced and have different amplitudes. However, the currents have the same amplitude and are approximately $120^{\circ}$ delayed. As previously stated, this figure helps to compute the voltage to current angle. Taking a look at the labels for the phase voltage $\mathrm{C}$, it indicates $134^{\circ}$, and the current angle shows $78^{\circ}$, corresponding to a difference of $134^{\circ}-78^{\circ}=56^{\circ}$ which strongly agrees with the theoretical impedance angle $\theta=55^{\circ}$. Therefore the proposed method ensures that the phase current can be injected in a particular angle with respect to the lowest phase voltage, in such a way that the voltage support is maximized.

\section{Impact of Grid Impedance Mismatches}

Last experiment also deals with the static analysis needed to further evaluate the optimal solution. Several tests have been developed using a wrongly estimated grid impedance, and the voltage support effects have been reported in Fig. 10. 


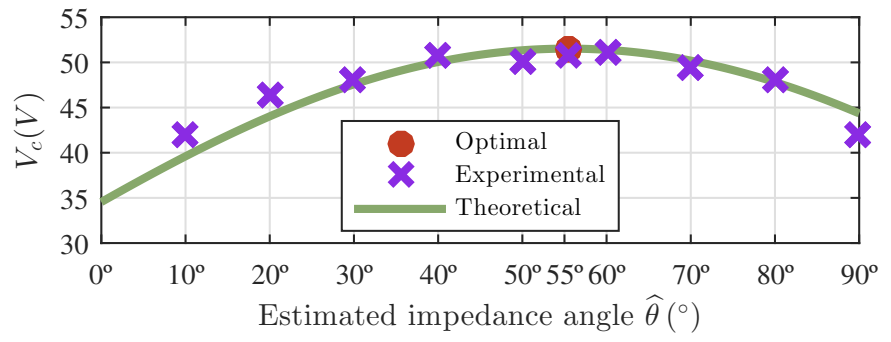

Fig. 10. Voltage support of phase $\mathrm{C}$ under impedance mismatch.

The figure shows the theoretical values according to (30) and (31) for the voltage in phase $\mathrm{C}$, and the experimental ones at time $t=0.1 \mathrm{~s}$. As shown in this plot, theoretic and experimental results agree (note that in both cases $V_{c}$ denotes peak voltage). It is worth mentioning that the test reveals that a perfectly known grid impedance is not really needed, since the voltage differences are low in a wide range of impedance values with respect to the optimal values.

Also, further analysis of Fig. 10 reveals the limitation of the conventional control for voltage support during voltage sags. Previous works assumed that the grid impedance was purely inductive, corresponding to the extreme-right part of this plot (i.e. $\theta=90^{\circ}$ ). With the proposed method that takes into account the resistive-inductive behavior of the grid, it is possible to improve the voltage in the lowest phase by approximately a $30 \%$ for the sag under test.

\section{CONCLUSIONS}

This paper has presented a voltage support control to improve the lowest phase voltage during grid faults. By supporting the lowest voltage, the main purpose of the proposal, which consists in reducing the risk of under-voltage disconnection is accomplished. A detailed mathematical formulation has been presented and a theoretical solution has been provided. This solution is valid for resistive and/or inductive grids, which widely expands the applicability of the voltage support from high to low and medium voltage systems, even in weak or stiff grids.

The experimental results have demonstrated the performance of the proposal even in a voltage sag with a complex dynamic profile. From these experiments, it can be concluded that the controller is capable of supporting the grid voltage while keeping the inverter safely operated during the fault, which is a must during these kind of perturbations.

Future work will be focused on the behavior of the proposal in a more complex grid, and the pros and cons for this method during other power events, including the islanding isolation of a part of the network, and the operation within microgrids.

\section{REFERENCES}

[1] T. Ackermann, G. Andersson, and L. Söder, "Distributed generation: a definition," Electric Power Syst. Research, no. 57, pp. 195-204, 2001.

[2] F. Blaabjerg, R. Teodorescu, M. Liserre, and A. V. Timbus, "Overview of control and grid synchronization for distributed power generation systems," IEEE Trans. Ind. Electron., vol. 53, no. 5, pp. 1398-1409, Oct. 2006
[3] S. Martin-Martinez, E. Gomez-Lazaro, A. Molina-Garcia, A. ViguerasRodriguez, M. Milligan, and E. Muljadi, "Participation of wind power plants in the spanish power system during events," in 2012 IEEE Power and Energy Soc. General Meeting, 2012, pp. 1-8.

[4] Red Eléctrica de España, Web resource, [Online, Accesed 1/29/2015], https://demanda.ree.es/eolicaEntreFechas.html.

[5] "New ERA for electricity in Europe. Distributed Generation: Key issuses, challenges and proposed solutions," European Commission, Energy Production and Distribution Systems, Rep., 2003.

[6] "IEEE Recommended Practice for Monitoring Electric Power Quality," IEEE Std. 1159-1995, 1995.

[7] "Distribution system power quality assessment: Phase II voltage sag and interruption analysis," EPRI. Electric Power Research Institute, Tech. Rep., Mar. 2003.

[8] M. Altin, O. Goksu, R. Teodorescu, P. Rodriguez, B. Bak-Jensen, and L. Helle, "Overview of recent grid codes for wind power integration," in 12th Int. Conf. on Optimization of Electrical and Electronic Equipment, May 2010, pp. 1152-1160.

[9] "Resolution-P.O.12.3-Response requirements against voltage dips in wind installations," Red Eléctrica de España (REE), Grid code, Oct. 2006.

[10] M. Tsili and S. Papathanassiou, "A review of grid code technical requirements for wind farms," IET Renewable Power Generation, vol. 3, no. 3, pp. 308-332, Sep. 2009.

[11] J. Jia, G. Yang, and A. H. Nielsen, "A review on grid-connected converter control for short circuit power provision under grid unbalanced faults," IEEE Trans. on Power Del., vol. PP, no. 99, pp. 1-1, 2017.

[12] H. C. Chen, C. T. Lee, P. T. Cheng, R. Teodorescu, and F. Blaabjerg, "A low-voltage ride-through technique for grid-connected converters with reduced power transistors stress," IEEE Trans. Power Electron., pp. 110, In press, 2016.

[13] J. L. Sosa, M. Castilla, J. Miret, J. Matas, and Y. A. Al-Turki, "Control strategy to maximize the power capability of pv three-phase inverters during voltage sags," IEEE Trans. Power Electron., vol. 31, no. 4, pp. 3314-3323, Apr. 2016.

[14] A. Milicua, G. Abad, and M. . R. Vidal, "Online reference limitation method of shunt-connected converters to the grid to avoid exceeding voltage and current limits under unbalanced operation; part i: Theory," IEEE Trans. Energy Conversion, vol. 30, no. 3, pp. 852-863, Sep. 2015.

[15] S. K. Chaudhary, R. Teodorescu, P. Rodriguez, P. C. Kjær, and A. Gole, "Negative sequence current control in wind power plants with VSCHVDC connection," IEEE Trans. Sustain. Energy, vol. 3, no. 3, pp. 535-544, Jul. 2012.

[16] X. Du, Y. Wu, S. Gu, H. M. Tai, P. Sun, and Y. Ji, "Power oscillation analysis and control of three-phase grid-connected voltage source converters under unbalanced grid faults," IET Power Electronics, vol. 9, no. 11, pp. 2162-2173, 2016.

[17] M. Mirhosseini, J. Pou, and V. G. Agelidis, "Individual phase current control with the capability to avoid overvoltage in grid-connected photovoltaic power plants under unbalanced voltage sags," IEEE Trans. Power Electron., vol. 30, no. 10, pp. 5346-5351, Oct 2015.

[18] Z. Dai, H. Lin, H. Yin, and Y. Qiu, "A novel method for voltage support control under unbalanced grid faults and grid harmonic voltage disturbances," IET Power Electron., vol. 8, no. 8, pp. 1377-1385, 2015.

[19] J. Miret, A. Camacho, M. Castilla, L. García de Vicuña, and J. de la Hoz, "Reactive current injection protocol for low-power rating distributed generation sources under voltage sags," IET Power Electronics, vol. 8, no. 6, pp. 879-886, 2015.

[20] A. Camacho, M. Castilla, J. Miret, R. Guzman, and A. Borrell, "Reactive power control for distributed generation power plants to comply with voltage limits during grid faults," IEEE Trans. Power Electron., vol. 29, no. 11 , pp. 6224-6234, Nov. 2014.

[21] W. Jiang, Y. Wang, J. Wang, L. Wang, and H. Huang, "Maximizing instantaneous active power capability for pwm rectifier under unbalanced grid voltage dips considering the limitation of phase current," IEEE Trans. Ind. Electron., vol. 63, no. 10, pp. 5998-6009, Oct 2016.

[22] X. Guo, X. Zhang, B. Wang, W. Wu, and J. M. Guerrero, "Asymmetrical grid fault ride-through strategy of three-phase grid-connected inverter considering network impedance impact in low-voltage grid," IEEE Trans. Power Electron., vol. 29, no. 3, pp. 1064-1068, Mar. 2014.

[23] A. Camacho, M. Castilla, J. Miret, P. Martí, and M. Velasco, "Maximizing positive sequence voltage support in inductive-resistive grids for distributed generation inverters during voltage sags," in 42th Ann. Conf. of IEEE Ind. Electron. Society, Oct 2016, pp. 2343-2348.

[24] F. Sulla, J. Svensson, and O. Samuelsson, "Wind turbines voltage support in weak grids," in 2013 IEEE Power Energy Soc. General Meeting, Jul. 2013, pp. 1-5. 
[25] P. Rodriguez, A. Luna, J. R. Hermoso, I. Etxeberria-Otadui, R. Teodorescu, and F. Blaabjerg, "Current control method for distributed generation power generation plants under grid fault conditions," in 37th Ann. Conf. on IEEE Ind. Electron. Society, Nov. 2011, pp. 1262-1269.

[26] J. A. Suul, A. Luna, P. Rodriguez, and T. Undeland, "Virtual-flux-based voltage-sensor-less power control for unbalanced grid conditions," IEEE Trans. Power Electron., vol. 27, no. 9, pp. 4071-4087, Sep. 2012.

[27] F. Wang, J. L. Duarte, and M. A. M. Hendrix, "Pliant active and reactive power control for grid-interactive converters under unbalanced voltage dips," IEEE Trans. Power Electron., vol. 26, no. 5, pp. 1511-1521, May 2011.

[28] S. Alepuz, S. Busquets-Monge, J. Bordonau, J. A. Martinez-Velasco, C. A. Silva, J. Pont, and J. Rodriguez, "Control strategies based on symmetrical components for grid-connected converters under voltage dips," IEEE Trans. Ind. Electron., vol. 56, no. 6, pp. 2162-2173, Jun. 2009.

[29] L. Asiminoaei, R. Teodorescu, F. Blaabjerg, and U. Borup, "Implementation and test of an online embedded grid impedance estimation technique for PV inverters," IEEE Trans. Ind. Electron., vol. 52, no. 4, pp. 11361144, Aug. 2005.

[30] A. Vidal, A. G. Yepes, F. D. Freijedo, O. López, J. Malvar, F. Baneira, and J. Doval-Gandoy, "A method for identification of the equivalent inductance and resistance in the plant model of current-controlled gridtied converters," IEEE Trans. Power Electron., vol. 30, no. 12, pp. 72457261, Dec 2015.

[31] F. J. Rodriguez, E. Bueno, M. Aredes, L. G. B. Rolim, F. A. S. Neves, and M. C. Cavalcanti, "Discrete-time implementation of second order generalized integrators for grid converters," in 34th Annu. Conf. of IEEE Ind. Electron., Nov. 2008, pp. 176-181.

[32] A. Yazdani and R. Iravani, Voltage-Sourced Converters in Power Systems. New Jersey, USA: Wiley-IEEE Press, 2010.

[33] A. Camacho, M. Castilla, J. Miret, A. Borrell, and L. García de Vicuña, "Active and reactive power strategies with peak current limitation for distributed generation inverters during unbalanced grid faults," IEEE Trans. Ind. Electron., vol. 62, no. 3, pp. 1515-1525, Mar. 2015.

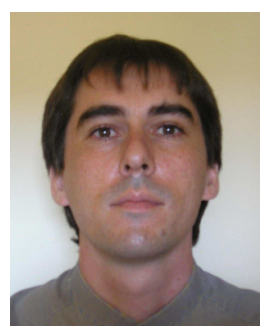

Antonio Camacho received the B.S. degree in chemical engineering, the M.S. degree in automation and industrial electronics, and the Ph.D. degree in electronic engineering, from the Technical University of Catalonia, Barcelona, Spain in 2000, 2009 and 2015 respectively.

His research interests include networked and embedded control systems, industrial informatics, and power electronics.

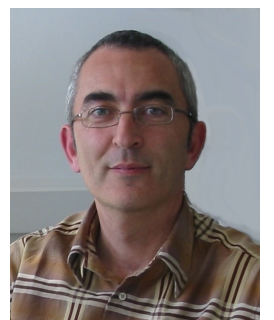

Miguel Castilla received the B.S., M.S. and $\mathrm{Ph}$.D. degrees in telecommunication engineering from the Technical University of Catalonia, Barcelona, Spain, in 1988, 1995, and 1998, respectively.

Since 2002, he has been an Associate Professor in the Department of Electronic Engineering, Technical University of Catalonia, where he teaches courses on analog circuits and power electronics. His research interests are in the areas of power electronics, nonlinear control, and renewable energy systems.

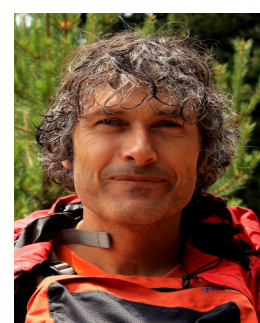

Jaume Miret (M'98) received the B.S. degree in telecommunications, M.S. degree in electronics, and Ph.D. degree in electronics from the Universitat Politecnica de Catalunya, Barcelona, Spain, in 1992, 1999, and 2005, respectively.

From 1993 to 2011, he was an Assistant Professor in the Department of Electronic Engineering, Universitat Politecnica de Catalunya, Spain. Since 2011 he has been an Associate Professor in the Universitat Politecnica de Catalunya, where he teaches courses on digital design and circuit theory. His research interests include dc-to-ac converters, active power filters, and digital control.

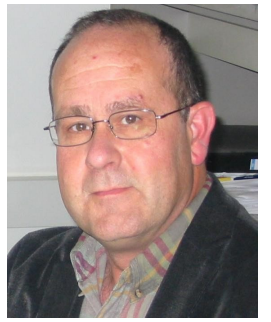

Luis García de Vicuña received the M.S. and Ph.D. degrees in Telecommunication Engineering from the Technical University of Catalonia, Barcelona, Spain, in 1980 and 1990, respectively, and the Ph.D. degree in Electrical Engineering from the Paul Sabatier University, Toulouse, France, in 1992.

$\mathrm{He}$ is currently a Full Professor with the Department of Electronic Engineering, Technical University of Catalonia, where he teaches courses on power electronics. His research interests include power electronics modeling, simulation and control, active power filtering, and high power-factor ac/dc conversion.

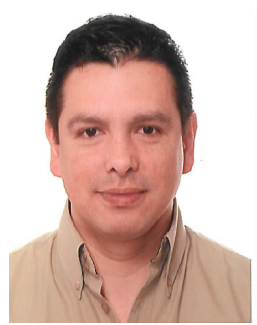

Miguel A. Garnica L. was born in San Juan de Pasto, Nariño, Colombia, in December 1973. $\mathrm{He}$ received the B.S. in naval engineering from Escuela Naval de Cadetes "Almirante Padilla", Cartagena de Indias, Colombia, in 2002 and the M.S. degree in electronic engineering from the University of Barcelona, Barcelona, Spain, in 2012.

$\mathrm{He}$ is currently working toward the Ph.D. degree in the Department of Electronic Engineering, Technical University of Catalonia, Spain. His research interests include power electronics, control systems, and renewable energy systems. 